\title{
Consequences of recent loophole-free experiments on a relaxation of measurement independence
}

\author{
Alejandro A. Hnilo* \\ CEILAP, Centro de Investigaciones en Láseres y Aplicaciones, UNIDEF (MINDEF-CONICET), CITEDEF, J. B. de La Salle 4397, \\ (1603) Villa Martelli, Argentina
}

(Received 11 August 2016; revised manuscript received 22 December 2016; published 2 February 2017)

\begin{abstract}
Recent experiments using innovative optical detectors and techniques have strongly increased the capacity of testing the violation of the Bell's inequalities in Nature. Most of them have used the Eberhard's inequality (EI) to close the "detection" loophole. Closing the "locality" loophole has been attempted by spacelike separated detections and fast changes of the bases of observation, driven by random number generators of new design. Also, pulsed pumping and time-resolved data recording to close the "time-coincidence" loophole, and sophisticated statistical methods to close the "memory" loophole, have been used. In this paper, the meaning of the EI is reviewed. A simple hidden variables theory based on a relaxation of the condition of "measurement independence," which was devised long ago for the Clauser-Horne-Shimony and Holt inequality, is adapted to the EI case. It is used here to evaluate the significance of the results of the recent experiments, which are briefly described. A table summarizes the main results.
\end{abstract}

DOI: 10.1103/PhysRevA.95.022102

\section{INTRODUCTION}

In 1965, Bell showed that the predictions of quantum mechanics (QM) are in contradiction with at least one of two intuitive notions; roughly speaking, (i) the result of a measurement cannot be affected by what happens outside its past light cone, and (ii) the existence of a world whose properties are independent of being observed [1]. The set of these two notions is usually named local realism (LR). The resolution of the QM vs LR controversy is crucial to the foundations of physics. Bell also proposed an experiment to decide whether QM or LR is valid in Nature, by measuring the violation of inequalities between the statistical averages of observations performed on a spatially spread entangled state of two particles.

Due to practical limitations, Bell's original proposal is difficult to perform. These limitations allow the existence of alternative descriptions, generally named hidden variable theories, which apparently violate the inequalities without violating LR. The types of practical limitations are known as loopholes [2]. The one often named locality or freedomof-choice loophole arises from the possibility that information is interchanged between the remote stations where the state is observed, and the source of entangled pairs. It implies the violation of the assumptions that the probability of joint detection is the product of the probabilities of detection at each station (locality) and that the hidden variables and the analyzers' settings in each station are statistically independent (measurement independence). To close this loophole, unpredictable and spacelike determined variation of the analyzers' settings must be achieved. The possibility that the state of the system varies in time, depending on earlier outcomes of the observations, is known as the memory loophole, and can be refuted through a special statistical analysis of the experimental data. The time-coincidence loophole is the possibility that the particle detections are shifted in time, in or out of the coincidence window. It can be disproved

\footnotetext{
*ahnilo@ citedef.gob.ar
}

also by a statistical analysis, but the simplest solution is to get a pulsed source of entangled pairs and a time-stamped record of the detections. Finally, the detection or fair-sampling loophole is the possibility that the particles are detected (or not) depending on the agreement of the hidden variables they carry with the analyzers' setting they find. To close it, detection efficiency higher than some threshold $\eta_{\text {thr }}$ must be achieved. For the Clauser-Horne-Shimony and Holt (CHSH) inequality, $\eta_{\mathrm{thr}}=2(\sqrt{2}-1) \approx 0.83,{ }^{1}$ for the Eberhard inequality (EI) $\eta_{\text {thr }}=2 / 3$. That is why the EI has been chosen in a series of recent optical experiments [3-6] aimed to reach the "loopholefree" condition.

In the next section, the derivation of the EI is reviewed. The QM predictions for the EI, which are not often available, are displayed. In Sec. III a hidden variables theory, which was devised long ago to evaluate experiments using the $\mathrm{CHSH}$, is adapted to the EI case. It defines a bound to the predictability of the analyzers' settings in order to close the locality loophole. The recent experiments using the EI and their main results are briefly described in Sec. IV. Another recent loophole-free experiment [7], which follows a different approach and uses $\mathrm{CHSH}$, is also described. Section $\mathrm{V}$ is the discussion of the consequences of the results of the five experiments, which are summarized in Table I.

\section{EBERHARD INEQUALITY}

The original form of the EI is [10]

$N^{++}(a, b)-N^{+0}\left(a, b^{\prime}\right)-N^{0+}\left(a^{\prime}, b\right)-N^{++}\left(a^{\prime}, b^{\prime}\right) \leqslant 0$,

where $N^{++}(i, j)$ is the number of coincidences recorded in an Einstein-Podolsky-Rosen-Bohm (EPRB) setup [1] when the analyzer's orientations settings are $\{i, j\}$, and $N^{+0}(i, j)\left[N^{0+}(i, j)\right]$ are the number of detections in station

\footnotetext{
${ }^{1} \eta_{\text {thr }}=0.83$ is the value stated in the earliest description of this loophole [8]; different approaches lead to $\eta_{\text {thr }}=1 / \sqrt{2} \approx 0.71$ and even to $[2(\sqrt{2}-1)]^{2} \approx 0.68$ [9]. The safest criterion is to use the most stringent condition.
} 
A (B) that do not produce coincidences. As the number of single detections in, e.g., station A is $S(a, j)=N^{+0}(a, j)+$ $N^{++}(a, j)$, it is possible to rewrite Eq. (1) as

$$
\begin{aligned}
& N^{++}(a, b)+N^{++}\left(a, b^{\prime}\right)+N^{++}\left(a^{\prime}, b\right)-N^{++}\left(a^{\prime}, b^{\prime}\right) \\
& \quad-S(a)-S(b) \leqslant 0,
\end{aligned}
$$

which is the Clauser-Horne $(\mathrm{CH})$ inequality [8]. This is the reason why the $\mathrm{EI}$ is often named the $\mathrm{CH}$-Eberhard inequality.

Ideally, Eq. (2) is violated by some entangled states, implying that QM is not compatible with LR. In order to violate the bound in a real experiment, the detection efficiencies must be taken into account. Assuming, for simplicity, that the efficiency $\eta$ is the same for all the detectors and settings, and dividing by the total number of pairs for each setting (assumed equal) in order to get probabilities, the Eq. (2) becomes

$$
\begin{aligned}
& \eta^{2}\left[P^{++}(a, b)+P^{++}\left(a, b^{\prime}\right)+P^{++}\left(a^{\prime}, b\right)-P^{++}\left(a^{\prime}, b^{\prime}\right)\right] \\
& -\eta\left[P^{+}(a)+P^{+}(b)\right] \equiv J \leqslant 0 .
\end{aligned}
$$

The probability of singles is always larger than the probability of coincidences for the same setting and, to make things even worse, singles are multiplied by $\eta$ instead of $\eta^{2}$, so that violating the inequality seems impossible for practical values of $\eta$.

Eberhard's brilliant idea was the use of a nonmaximally entangled state. It is certainly anti-intuitive that, to test QM vs LR, a partially entangled state can be better than a maximally entangled one. He defined the state $(r<1)$ as follows:

$$
\left|\psi_{E}\right\rangle=\left(1+r^{2}\right)^{-1 / 2}\left\{\left|x_{A}, y_{B}\right\rangle+r\left|y_{A}, x_{B}\right\rangle\right\}
$$

whose concurrence is $2|r| /\left(1+r^{2}\right)$. The probability of coincidences for this state is

$$
P^{++}(a, b)=\left(1+r^{2}\right)^{-1}[\cos (a) \sin (b)+r \sin (a) \cos (b)]^{2},
$$

and the probabilities of single detections are as follows:

$$
\begin{aligned}
& P^{+}(a)=\left(1+r^{2}\right)^{-1}\left[\cos ^{2}(a)+r^{2} \sin ^{2}(a)\right], \\
& P^{+}(b)=\left(1+r^{2}\right)^{-1}\left[r^{2} \cos ^{2}(b)+\sin ^{2}(b)\right] .
\end{aligned}
$$

Note that $P^{+}(a) \neq \frac{1}{2} \neq P^{+}(b)$. In other words, the photons in each station are observed as partially polarized. Equations. (4)-(7) converge to the usual case if $r=1$.

The state $\left|\psi_{E}\right\rangle$ is able to violate Eq. (3) for lower values of $\eta_{\text {thr }}$ in the following way: Choosing the angle settings so that $\cos (a) \approx 0$ and $\sin (b) \approx 0$, the single probabilities are $\approx r^{2}$. From Eq. (5) also $P^{++}(a, b), P^{++}\left(a, b^{\prime}\right)$, and $P^{++}\left(a^{\prime}, b\right) \approx r^{2}$. The settings $\left\{a^{\prime}, b^{\prime}\right\}$ are still free to make $P^{++}\left(a^{\prime}, b^{\prime}\right) \approx 0$. Then

$$
J \approx 3 \eta r^{2}-2 r^{2} \leqslant 0,
$$

so that $\eta>2 / 3$ to violate the bound, which is the well-known result. Note that the key is the small value $\left(\approx r^{2}\right)$ of the probabilities of single detection, thanks to the wise choosing of the (partially entangled) state emitted by the source and the almost nontransmitting orientation of the analyzers. Fine tuning of $r$ and the angle settings with a computer code maximizes $J$.

Replacing Eqs. (5)-(7) into Eq. (3) gives the ideal QM prediction value $J_{\mathrm{QM}}$. It is easy to calculate, but cumbersome

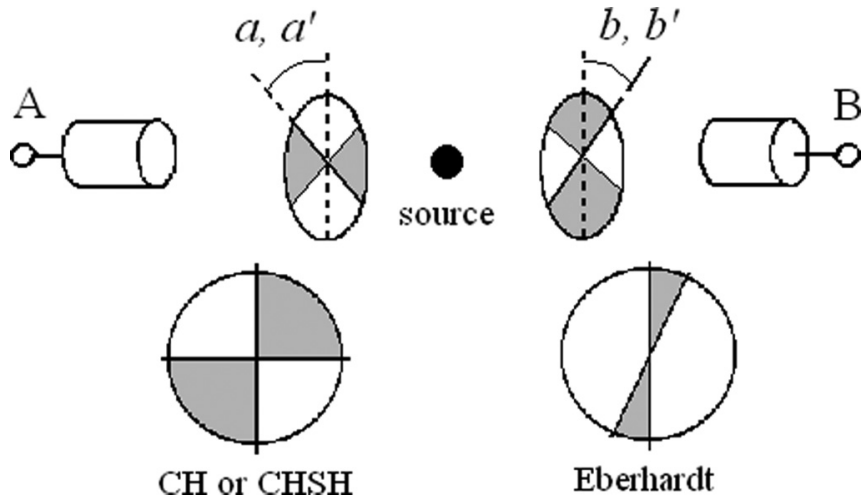

FIG. 1. Each analyzer has transmitting (gray) and reflective (white) regions in the space of the hidden variable $\lambda$. The probability of coincident detection is simply given by the overlap of the transmitting regions. The regions' sizes are different if $r=1(\mathrm{CH}$ or CHSH, $P^{+}=\frac{1}{2}$ ) or $r<1$ (EI, typically $P^{+}<0.1$ ).

to display as an explicit function. An approximate expression is

$$
J_{\mathrm{QM}} \approx \eta r^{2} \text {. }
$$

For example, for the values in [4] and $\eta=1$, the exact value of $J_{\mathrm{QM}}$ is 0.067 , while the one from Eq. (9) is 0.084 .

Some setups use the state $\left|\phi_{E}\right\rangle=\left(1+r^{2}\right)^{-1 / 2}\left\{\left|\mathrm{x}_{\mathrm{A}}, \mathrm{x}_{\mathrm{B}}\right\rangle+\right.$ $\left.\mathrm{r}\left|\mathrm{y}_{\mathrm{A}}, \mathrm{y}_{\mathrm{B}}\right\rangle\right\}$ instead of $\left|\psi_{E}\right\rangle$. The coincidence probability is

$$
P^{++}(a, b)=\left(1+r^{2}\right)^{-1}[\cos (a) \cos (b)+r \sin (a) \sin (b)]^{2},
$$

while the probability of single detections is given by Eq. (6) for both stations, due to the symmetry of $\left|\phi_{E}\right\rangle$.

\section{HV + DZ FOR THE EBERHARD INEQUALITY}

In 1991, I proposed a simple model called "hidden variables with directionalization" ( $\mathrm{HV}+\mathrm{DZ}$ ) as a tool to evaluate the results of experiments using the $\mathrm{CH}$ or $\mathrm{CHSH}$ inequalities [11].

To make this paper self-consistent, the $\mathrm{HV}+\mathrm{DZ}$ model is reviewed in the Appendix. In brief, assume a yes-no probability of passage on a hidden variable $\lambda$ (see Fig. 1). This scheme saturates the inequality. It may seem that a small shift of the transmitting regions suffices to violate it. But it does not, because the advantage obtained for some analyzers' settings is compensated by the disadvantage for others. A hidden variable $\mu$ is then added, that defines "target" settings for the particles. A critical parameter is the correlation $q\left(q>\frac{1}{2}\right)$ between the value of $\mu$ and the settings. This correlation means a violation of the condition of measurement independence. The experiments attempt to enforce it by random and spacelike choosing of the settings. Yet, some (small) correlation may still exist due to the statistical predictability of the future settings (because of some unbalance or bias in their distribution) and the instrumental imperfections. The problem addressed by $\mathrm{HV}+\mathrm{DZ}$ is to calculate the minimum degree of correlation necessary to reproduce the QM predictions.

$\mathrm{HV}+\mathrm{DZ}$ was devised to study the experiment by Aspect et al. [12]. Later, it was used to evaluate the improvements reached by the experiment by Weihs et al. [13,14]. The correlation necessary to reproduce the ideal QM predictions for 


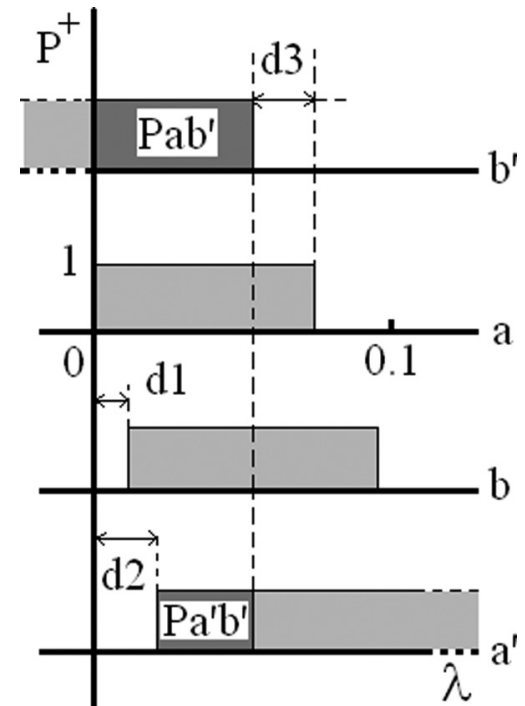

FIG. 2. Scheme of the probability distributions for each setting and station as a function of the hidden variable $\lambda$, for $\mathrm{HV}+\mathrm{DZ}$ adapted to the EI. Light gray: transmission regions. Dark gray: two regions of coincidence, indicated as an illustration. The horizontal axis is scaled such that it directly reads the probability of passage.

the $\mathrm{CH}$ and $\mathrm{CHSH}$ inequalities is $q=\left(1 / \sqrt{2}-\frac{7}{16}\right)^{1 / 2}+\frac{1}{4} \approx$ 0.769 , or $\approx 0.27$ in excess to the minimum value of $\frac{1}{2}$. The consequences of a deviation from perfect measurement independence were considered by several authors from different points of view [15-17]. A general approach almost halves the excess value calculated in $\mathrm{HV}+\mathrm{DZ}$, to $(\sqrt{2}-1) / 3 \approx$ 0.14 [15].

In summary: a relatively small relaxation of measurement independence allows reproducing the ideal QM predictions for the $\mathrm{CH}$ and $\mathrm{CHSH}$ inequalities. It is then pertinent to study how such relaxation affects the EI.

To adapt HV + DZ to the EI, the size of the "transmitting" regions is given by Eqs. (6) and (7). Typically, $P^{+}(a), P^{+}(b)<0.1$, and $P^{+}\left(a^{\prime}\right), P^{+}\left(b^{\prime}\right) \approx \frac{1}{4}$. The resultant probability distributions on $\lambda$ for each station and setting are sketched in Fig. 2 (compare with Fig. 3 in the Appendix). The gray regions have height equal to 1 (indicating probability $=1$ of transmission for the corresponding values of $\lambda$ ) and their widths are scaled here to be equal to the probabilities of single passage. For example, using the data in [5] the lengths of the areas in the axes named $a$ and $b$ are $P^{+}(a)=0.0825$ and $P^{+}(b)=0.0884$. The values of $P^{+}\left(a^{\prime}\right)$ and $P^{+}\left(b^{\prime}\right)$ extend out of the figure and are irrelevant for the EI. The overlap of two areas gives the probability of the corresponding coincidence. In Fig. 2, the dark gray areas indicate $P^{++}\left(a, b^{\prime}\right)$ and $P^{++}\left(a^{\prime}, b^{\prime}\right)$ as an illustration. The distances $d_{j}$ are defined as

$$
\begin{aligned}
P^{++}(a, b) & =P^{+}(a)-d_{1}, \\
P^{++}\left(a^{\prime}, b\right) & =P^{+}(b)+d_{1}-d_{2}, \\
P^{++}\left(a, b^{\prime}\right) & =P^{+}(a)-d_{3}, \\
P^{++}\left(a^{\prime}, b^{\prime}\right) & =P^{+}(a)-d_{2}-d_{3},
\end{aligned}
$$

the EI is saturated $(J=0)$ regardless of the values of the $d_{j}$.

Let us define now an auxiliary hidden variable $\mu$. The pairs with $\mu=1$ have the setting $\{a, b\}$ as their target; the ones with $\mu=2$ the $\left\{a, b^{\prime}\right\}, \mu=3$ the $\left\{a^{\prime}, b\right\}$, and $\mu=4$ the $\left\{a^{\prime}, b^{\prime}\right\}$. Each pair of emitted particles tries to reach its target by guessing the future settings when leaving the source, and also by exploiting the instrumental imperfections when the guess fails. The probability $q$ of reaching the target is (at first order) the sum of the probabilities of success of both strategies. In general, $q$ measures the correlation between $\mu$ and the settings regardless of the physical cause; $\varepsilon=q-\frac{1}{2}>0$ is then the deviation from perfect measurement independence. As all the numbers in the EI are smaller than in CHSH the necessary shifts (see the Appendix) are also smaller, and besides, they are different for each setting. The set of the $d_{j}$ (in CHSH are all equal to $\pi / 8$ ) is therefore enlarged to a set of $d_{j \mu}$ whose values are chosen to maximize the value of $J$ :

$$
\begin{aligned}
P^{\mu=1}(a, b) & =P^{+}(a)\left(\Leftrightarrow d_{11}=0\right), \\
P^{\mu=2}\left(a, b^{\prime}\right) & =P^{+}(a)\left(\Leftrightarrow d_{32}=0\right), \\
P^{\mu=3}\left(a^{\prime}, b\right) & =P^{+}(b)\left(\Leftrightarrow d_{13}=d_{23}\right), \\
P^{\mu=4}\left(a^{\prime}, b^{\prime}\right) & =0\left[\Leftrightarrow d_{24}+d_{34}=P^{+}(a)\right] .
\end{aligned}
$$

For example, for $\mu=1$ one wants to get $P^{\mu=1}(a, b)=$ $P^{+}(a)$, which is the maximum possible value for $P^{++}(a, b)$. Therefore, one must define $d_{11}=0$, and then it follows that $P^{\mu=1}\left(a, b^{\prime}\right)=P^{+}(a)-d_{31}, P^{\mu=1}\left(a^{\prime}, b\right)=P^{+}(b)-d_{21}$, and $P^{\mu=1}\left(a^{\prime}, b^{\prime}\right)=P^{+}(a)-d_{21}-d_{31}$. In the same way, expressions for all the $P^{\mu}(i, j)$ are found. Note that the values of some $d_{j \mu}$ remain free. Assuming for simplicity that the correlation $q$ is the same for all $\mu$, the observable coincidence probabilities are

$$
\begin{aligned}
P^{++}(a, b) & =q^{2} P^{\mu=1}(a, b)+q(1-q)\left[P^{\mu=2}(a, b)+P^{\mu=3}(a, b)\right]+(1-q)^{2} P^{\mu=4}(a, b), \\
P^{++}\left(a, b^{\prime}\right) & =q^{2} P^{\mu=2}\left(a, b^{\prime}\right)+q(1-q)\left[P^{\mu=1}\left(a, b^{\prime}\right)+P^{\mu=4}\left(a, b^{\prime}\right)\right]+(1-q)^{2} P^{\mu=3}\left(a, b^{\prime}\right), \\
P^{++}\left(a^{\prime}, b\right) & =q^{2} P^{\mu=3}\left(a^{\prime}, b\right)+q(1-q)\left[P^{\mu=1}\left(a^{\prime}, b\right)+P^{\mu=4}\left(a^{\prime}, b\right)\right]+(1-q)^{2} P^{\mu=2}\left(a^{\prime}, b\right), \\
P^{++}\left(a^{\prime}, b^{\prime}\right) & =q^{2} P^{\mu=4}\left(a^{\prime}, b^{\prime}\right)+q(1-q)\left[P^{\mu=2}\left(a^{\prime}, b^{\prime}\right)+P^{\mu=3}\left(a^{\prime}, b^{\prime}\right)\right]+(1-q)^{2} P^{\mu=1}\left(a^{\prime}, b^{\prime}\right) .
\end{aligned}
$$

Replacing the $P^{\mu}(i, j)$ of Eq. (12) into Eq. (13) and then into Eq. (3) with $\eta=1$, the value of $J$ according to the HV + DZ model is

$$
\begin{aligned}
J_{\mathrm{DZ}}= & q^{2} P^{+}(a)-(1-q)\left\{q P^{+}(a)+(1-2 q)\left[d_{14}+d_{22}+d_{33}-\left(d_{12}+d_{21}+d_{31}\right)\right]\right\} \\
= & q^{2} P^{+}(a)-q(1-q) P^{+}(a)-(1-q)(1-2 q)\left\{P^{+}(a)+P^{+}(b)-P^{\mu=4}(a, b)-P^{\mu=2}\left(a^{\prime}, b\right)\right. \\
& \left.-P^{\mu=3}\left(a, b^{\prime}\right)+P^{\mu=1}\left(a^{\prime}, b^{\prime}\right)\right\} .
\end{aligned}
$$


Note that $q=\frac{1}{2} \Rightarrow J_{\mathrm{DZ}}=0$, as it must be. The factor between keys in the second equality has the form of an EI but with the opposite sign and coincidence probabilities for nontarget settings. It is convenient to define this factor as $\left(-J^{\prime}\right)$, so that Eq. (14) is written

$$
J_{\mathrm{DZ}}=q^{2} P^{+}(a)-q(1-q) P^{+}(a)+(1-q)(2 q-1)\left(-J^{\prime}\right) \text {. }
$$

The numerical value of $J^{\prime}$ depends on the precise choosing of the $d_{j \mu}$ that remained free. It is always $J^{\prime} \leqslant 0$ because the choosing defines a LR theory, which necessarily holds to the EI. The last term in Eq. (15) is hence positive, or zero. Then

$$
J_{\mathrm{DZ}} \geqslant P^{+}(a)\left(\varepsilon+2 \varepsilon^{2}\right)
$$

so that the EI is violated by any $q>\frac{1}{2}$. The same is valid for the original $\mathrm{HV}+\mathrm{DZ}$ case $(r=1)$ and $\mathrm{CHSH}$ :

$$
S=2\left(1+\varepsilon+2 \varepsilon^{2}\right) \text {. }
$$

A different situation is faced if $J$ is required not to merely violate the bound, but to fit the QM prediction $J_{\mathrm{QM}}$. For a choosing of the remaining $d_{j \mu}$ such that $-J^{\prime}=2 P^{+}(a)+$ $P^{+}(b) \approx 3 P^{+}(a)$ then, from Eq. (15),

$$
q_{\mathrm{QM}} \approx 1-\frac{1}{2}\left[1-J_{\mathrm{QM}} / P^{+}(a)\right]^{1 / 2}
$$

for the ideal values in [5]; $q_{\mathrm{QM}} \approx 0.78$ (compare with $q_{\mathrm{QM}} \approx$ 0.769 required by the original $\mathrm{HV}+\mathrm{DZ}$ model to fit $S_{\mathrm{CHSH}}=$ $2 \sqrt{2})$. Finally, replacing the measured values of $J$ and $P^{+}(a)$ in Eq. (18), the minimum correlation $q_{m}$ needed to reproduce the observed violation of the EI is obtained.

In summary: the counterexample provided by the $\mathrm{HV}+$ DZ model shows that the EI bound is violated as soon as a relaxation of measurement independence is allowed, even if $\eta=1$. The same holds for the CHSH. The ideal values $J_{\mathrm{QM}}$ and $S=2 \sqrt{2}$ are reached by $q_{\mathrm{QM}} \approx \frac{3}{4}$ in both cases. Hence, the capacity to discriminate QM from LR is the same by the EI and the CHSH. This result concurs with the conclusions reached in $[18,19]$ from a different point of view.

\section{BRIEF DESCRIPTION OF THE RECENT EXPERIMENTAL RESULTS}

\section{A. Definition of relevant parameters in common}

Three experiments [3-5] (see Secs. IV B-IV D) are based on the use of transition edge sensors (TESs) [20] operating at cryogenic temperatures. These photon detectors have a separately measured efficiency up to $98 \%$, and are crucial to closing the detection loophole.

In order to close the memory loophole, a refined statistical method is performed, which was first developed in [21]. The idea is to calculate the maximal probability (called the $p$ value) that the observed outcome has been produced by a statistical fluctuation under the conditions to be disproved, in this case, LR. This type of test was formalized for the first time in [22,23]. Other methods to calculate the $p$ value were developed in [24-26], to include a bias in the setting distributions (which is a common practical imperfection) for both the $\mathrm{CHSH}$ and EI. The predictability of the settings is taken into account in a recently published paper [27], allowing for two different cases: perfect predictability in some trials, or else, constant average predictability during the whole run. The latter can be considered as a different approach to the situation addressed by HV + DZ. The two approaches lead to the same conclusion. The $p$ value is used in the experiments [5-7] detailed in Secs. IV D-IV F to close the memory loophole, and in Secs. IV D and IV E [5,6] also as an alternative to the calculation of the standard error of $J$ to quantify the reliability of the results. A main practical concern is finding the value of the sample size (or cut point) that provides a statistically significant $p$ value. This can be done without assuming any a priori distribution, following a special procedure that is detailed, e.g., in the Supplemental Material section of the experiment [6] (Sec. IV E). Note that the value $q_{m}$ obtained from $\mathrm{HV}+\mathrm{DZ}$ is not the consequence of a statistical fluctuation, but of a systematical behavior at the hidden variables level. The results of $\mathrm{HV}+\mathrm{DZ}$ are valid even in the limit of an infinite statistical set. In general, the $p$ value and $q_{m}$ define different and complementary bounds.

In principle, the correlation between the hidden variable $\mu$ and the analyzers' settings can have two causes: the predictability of the settings and the instrumental imperfections of the settings' realizations. In the originally studied experiment of Aspect et al., the latter was mainly determined by the contrast of the acousto-optical modulators that deflected the beams towards the different fixed settings. In the recent experiments, it is related with the latency time of the electro-optical modulators (EOMs) and the errors in the time-stamping devices. For simplicity, I take into account here the first cause only. The predictability of the settings is given mainly by the bias of the random number generators (RNGs). Using physical models of the random processes and measurements of the RNG outputs, estimates of the predictability in each setup, $q_{\text {set }}$, can be developed. In what follows, the values of $q_{\text {set }}$ are the ones estimated by the authors of the experiments. In order to close the locality loophole, the value of $q_{\text {set }}$ must be smaller than the value $q_{m}$ the $\mathrm{HV}+\mathrm{DZ}$ needs to reproduce the observed value $J_{m}$.

Other relevant parameters are the separately measured efficiencies of the detectors $\eta_{\text {meas }}$. Inserted in Eq. (3), they provide a "first-order" corrected value $J_{\text {corr }}$. One expects $J_{\text {corr }} / J_{m} \approx 1$. Conversely, $\eta_{\text {eq }}$ is the efficiency value that, inserted into Eq. (3) with the ideal values of the probabilities, reproduces $J_{m}$. It is a compact way to take into account the experimental imperfections. Ideally, $\eta_{\mathrm{eq}} \approx \eta_{\text {meas }}$. The criterion to close the detection loophole is $\eta_{\mathrm{eq}}>\eta_{\mathrm{thr}}$. Recall that, in the experiments using the EI, $\eta_{\text {thr }}$ depends on the level of noise.

Be aware that the CHSH parameter $S$ is used instead of $J$ in the experiment in Sec. IV F. Also, note that the coincidence rate depends on the angle settings, which are different in each experiment, so that a direct comparison is impossible. Hence, the values displayed in Table I just give a rough idea of the rate of detected particles and of the contrast of the coincidence vs angle curves in each case.

\section{B. Giustina et al. [3]}

A continuous wave (cw) laser diode at $405 \mathrm{~nm}$ pumps a ppKTP-II crystal placed inside a Sagnac interferometer. The insertion of additional crystals on the pump beam prepares the state $\left|\psi_{E}\right\rangle$ with $r \approx 0.3$. The pairs of photons at $810 \mathrm{~nm}$ are selected with interferential filters, focused into single-mode 
TABLE I. Summary of some parameters of interest; $J_{m}$ is the experimentally obtained value of the left-hand side of the EI, $J_{\mathrm{QM}}$ is the QM ideal prediction, $J_{\text {corr }}$ is the QM prediction but corrected by the separately measured efficiencies $\eta_{\text {meas }}, \eta_{\text {eq }}$ is the efficiency value that makes $J_{\text {corr }}=J_{\mathrm{m}}, q_{\mathrm{QM}}$ is the correlation probability between the hidden variable $\mu$ and the analyzers' settings the $\mathrm{HV}+\mathrm{DZ}$ needs to reproduce $J_{\mathrm{QM}}$, $q_{m}$ is the same but to reproduce $J_{m}$, and $q_{\text {set }}$ is the predictability of the RNG as estimated by the authors of the experiments.

\begin{tabular}{|c|c|c|c|c|c|}
\hline & $\begin{array}{l}\text { Giustina et al. } \\
\text { [3]; see Sec. IV B }\end{array}$ & $\begin{array}{l}\text { Christensen } \text { et al. } \\
\text { [4]; see Sec. IV C }\end{array}$ & $\begin{array}{l}\text { Giustina et al. } \\
\text { [5]; see Sec. IV D }\end{array}$ & $\begin{array}{l}\text { Shalm et al. } \\
\text { [6]; see Sec. IV E }\end{array}$ & $\begin{array}{l}\text { Hensen } \text { et al. } \\
\text { [7]; see Sec. IV F }\end{array}$ \\
\hline$J_{m}$ & $5.24 \times 10^{-3} \pm 8 \times 10^{-5}$ & $5.4 \times 10^{-5} \pm 7 \times 10^{-6}$ & $7.27 \times 10^{-6}$ & $1.41 \times 10^{-5}$ & $S_{m}=2.42 \pm 0.07$ \\
\hline$J_{\mathrm{QM}}$ & 0.0701 & 0.0549 & 0.0671 & 0.0645 & $S_{\mathrm{QM}}=2 \sqrt{2}$ \\
\hline$J_{\text {corr }}$ & $8.53 \times 10^{-3}$ & $5.7 \times 10^{-2}$ & $1.03 \times 10^{-2}$ & $6.26 \times 10^{-3}$ & $S_{\text {corr }}=2.30$ \\
\hline$\eta_{\text {meas }}$ & 0.738 and 0.786 & 0.75 & 0.786 and 0.762 & 0.747 and 0.756 & 0.971 and 0.963 \\
\hline$\eta_{\mathrm{eq}}$ & 0.745 & 0.710 & 0.719 & 0.715 & Not applicable \\
\hline$\eta_{\mathrm{thr}}$ & 0.667 & 0.667 & $0.667(?)$ & 0.725 & 0.828 \\
\hline$J_{\text {corr }} / J_{m}$ & 1.6 & 106 & 1422 & 444 & 0.95 \\
\hline$q_{\mathrm{QM}}$ & 0.785 & 0.787 & 0.783 & 0.776 & 0.769 \\
\hline$q_{m}$ & $\frac{1}{2}+2.1 \times 10^{-2}$ & $\frac{1}{2}+8 \times 10^{-3}$ & $\frac{1}{2}+7.6 \times 10^{-3}$ & $\frac{1}{2}+1.6 \times 10^{-2}$ & 0.659 \\
\hline$q_{\mathrm{set}}$ & 1 & 1 & $\frac{1}{2}+1.2 \times 10^{-4}$ & $\frac{1}{2}+10^{-4}$ & $\frac{1}{2}+10^{-5}$ \\
\hline$p$ Value & Not computed & $1.16 \times 10^{-10}[32]$ & $3.74 \times 10^{-31}$ & $5.9 \times 10^{-9}$ & 0.039 \\
\hline Coincidence rate (min) & $232 \mathrm{~s}^{-1}$ & $1.7 \mathrm{~s}^{-1}$ & $9.6 \mathrm{~s}^{-1}$ & $0.059 \mathrm{~s}^{-1}$ & $\approx 2 \times 10^{-5} \mathrm{~s}^{-1}$ \\
\hline Coincidence rate (max) & $3970 \mathrm{~s}^{-1}$ & $31 \mathrm{~s}^{-1}$ & $162 \mathrm{~s}^{-1}$ & $3.60 \mathrm{~s}^{-1}$ & $\approx 1.3 \times 10^{-4} \mathrm{~s}^{-1}$ \\
\hline
\end{tabular}

optical fibers; they find analyzers with fixed settings, and are detected with TESs. The time of detection of each photon is stored in a time-tagged file. Each setting is left fixed during a period of $300 \mathrm{~s}$.

The measured value of the violation of the EI is $J_{m}=$ $5.24 \times 10^{-3} \pm 8 \times 10^{-5}$, while the ideal value is $J_{\mathrm{QM}}=$ $7.01 \times 10^{-2}$. The difference is explained by taking into account several practical imperfections [26]. Without going into the details, note that $J_{\text {corr }}=8.53 \times 10^{-3}$, which is reasonably close to $J_{m}$. Conversely, the equivalent efficiency is $\eta_{\text {eq }}=0.745$. Hence, assuming a small deterioration of the average efficiency suffices to explain the difference between $J_{m}$ and $J_{\text {corr. }}$. This feature is found also in the other experiments using EI, where $J_{\text {corr }} / J_{m}$ is even larger (see Table I). The reason why the value of the efficiency is so critical to the value of $J$ is a consequence of the EI, which deals with numbers that are all close to zero.

The measured value of $P^{+}(a)$ is 0.063 , reasonably close to the ideal 0.088. From Eq. (18), $q_{m} \approx \frac{1}{2}+2 \times 10^{-2}$. In this setup and the next the settings are fixed, so that $q_{\text {set }}=1>q_{m}$. Recall, however, that these experiments were not aimed to close the locality loophole.

\section{Christensen et al. [4]}

The third harmonic of a mode-locked Nd:YAG laser (5 ps at $120 \mathrm{MHz}$ ) is used to pump a pair of crossed BBO-I crystals in a standard configuration. Additional crystals prepare the state $\left|\phi_{E}\right\rangle$ with $r=0.26$. The pairs of photons at $710 \mathrm{~nm}$ are filtered with single-mode optical fibers and detected with a TES cooled at $100 \mathrm{mK}$. The TESs have a jitter of about $1 \mu \mathrm{s}$, so that they are unable to resolve the time between the mode-locking pulses. The pump beam is then modulated with a Pockels cell to produce bursts $1 \mu$ s long (or 240 mode-locking pulses), separated by $40 \mu \mathrm{s}$. The time of detection of each photon is saved in a time-tagged file. The settings are randomly changed with a periodicity of $1 \mathrm{~s}$. This is not done to close the locality loophole (the period is too long for that), but to avoid any instrumental drift that may produce a spurious violation of the EI.

The measured value $J_{m}=5.4 \times 10^{-5} \pm 7 \times 10^{-6}$ is three orders of magnitude smaller than the ideal $J_{\mathrm{QM}}=5.49 \times$ $10^{-2}$, yet $\eta_{\mathrm{eq}}=0.71$ suffices to fit the two values. In this experiment and in the previous one $\eta_{\mathrm{eq}}>\eta_{\mathrm{thr}}=2 / 3$, so that the detection loophole is successfully closed in both cases. In this experiment the $\mathrm{CHSH}$ parameter is also measured (after adjusting $r=1$ ) and the excellent value $S_{\mathrm{CHSH}}=2.827 \pm$ 0.017 is obtained.

The measured value of $P^{+}(a)$ is $\approx 1.69 \times 10^{-3}$, much smaller than the ideal 0.067 . However, as $J_{m}$ is also small, $q_{m}$ is only $\approx \frac{1}{2}+8 \times 10^{-3}$.

\section{Giustina et al. [5]}

The source is the same as in [3] (Sec. IV B) (with $r=$ -0.29 ) but the 405-nm pump laser diode is now modulated to emit pulses $12 \mathrm{~ns}$ FWHM at $1 \mathrm{MHz}$ repetition rate. An output signal from the laser synchronizes the measuring process and defines the "natural time" a valid photon is expected to arrive to the stations. The entangled photons are spectrally and spatially filtered by focusing into single-mode optical fibers, which transport them to the stations of observation. The stations are separated $\approx 58 \mathrm{~m}$, with the source near the middle point. In each station, EOMs are driven to change the angle settings of the analyzers. The settings are decided by identical RNGs, one in each station. The RNGs are based on laser phase diffusion, and produce raw series of random bits at $200 \mathrm{MHz}$ speed. At the time an output is required, only the most recent raw bits are chosen to run a parity calculation to decide the measurement setting. This is to make sure that the definitions of the settings are spacelike separated. The process of choosing and driving the EOM is completed in only $26 \mathrm{~ns}$, shorter than the distance from each station to the source $(\approx 87 \mathrm{~ns})$. The predictability of this process is estimated smaller than $\frac{1}{2}+1.2 \times 10^{-4}$. The photons are detected with 
TES with separately measured efficiencies $\eta_{\mathrm{A}}=0.786$ and $\eta_{\mathrm{B}}=0.762$.

All the relevant data are saved in time-tagged files. The digitizer operates in a triggered mode starting with a photon detected during the natural time (which is defined by the signal coming from the pump laser), but it also records $1024 \mathrm{~ns}$ before and after the trigger at a sample rate of $250 \mathrm{MHz}$, or $4 \mathrm{~ns}$ time resolution. This is far more than enough, taking into account the jitter of the detectors. One limitation is that the digitizer requires $2.176 \mu$ s to rearm after a trigger. This means that it is blind to the next two or three pump pulses. Yet, this is not a serious limitation, for the probability of producing one photon per pulse is low, to keep the number of accidental coincidences small [28].

Data are recorded during $4.8 \mathrm{~h}$, in three blocks of 1,1 , and $2.8 \mathrm{~h}$. The experiment stops when a real-time running check of entanglement indicates that the setup is drifting out of alignment. Using the second block of data, the value $J_{m}=$ $7.27 \times 10^{-6}$ is obtained, four orders of magnitude smaller than the ideal one.

The measured value of $P^{+}(a)$ is not directly provided. As the singles in station 1 must be independent of the setting in station 2 , I estimate $P^{+}(a)$ from the sum $N_{11}^{++}+N_{12}^{+0}=$ $141439+67941$ (see Supplemental Material in [5]) and dividing by the average of the number of trials in each case, or $(875683790+875518074) / 2$, then $P^{+}(a) \approx 2.4 \times 10^{-4}$. It agrees with the value $2.2 \times 10^{-4}$ obtained using $\eta_{\mathrm{eq}}$, but it is far from the ideal 0.083. Anyway, $q_{m}=\frac{1}{2}+7.6 \times 10^{-3}$ is larger than $q_{\text {set }}=\frac{1}{2}+1.2 \times 10^{-4}$, so that the locality loophole is closed. If the ideal value of $P^{+}(a)$ were used instead, $q_{m}=\frac{1}{2}+2.2 \times 10^{-5}$ and the loophole would not be closed. The $p$ value defined in this experiment takes into account the predictability of the settings. It is measured smaller than $3.74 \times 10^{-31}$, hence closing both the memory and the locality loopholes.

Background photons raise the value of $\eta_{\mathrm{thr}}$. Here, the electrical signal produced by the TES is digitized and its shape is used to discriminate detections of valid photons at $810 \mathrm{~nm}$ from photons of lower energy coming from blackbody radiation. However, remaining background is claimed, together with imperfect state purity, for the large difference between $J_{m}$ and $J_{\text {corr }}$. I have found no reported estimation of the remaining background level or of the increase of $\eta_{\mathrm{thr}}$.

\section{E. Shalm et al. [6]}

A mode-locked Ti:sapphire laser emits ps pulses at a repetition rate of $79.3 \mathrm{MHz}$ at $775 \mathrm{~nm}$. It is split into two beams with orthogonal polarizations to pump a ppKTP crystal. These two beams are inserted into a polarization Mach-Zehnder interferometer that allows preparing the state $\left|\phi_{E}\right\rangle(r=0.287)$. The downconverted photons are in the communications band, which is an attractive feature of this setup. They are sent to the observation stations via optical fibers. The mode-locked pulses are used as a clock to synchronize the measurements. They are detected at the laser's output with a fast photodiode, one each of 800 is picked out, and the electrical signal is sent to the stations. Once per second, a signal from the GPS helps to prevent any slow drift between the time tags in each station during an experimental run (30 min).
The source and the stations are positioned at the vertices of a nearly right-angle triangle. Each station is at about $130 \mathrm{~m}$ from the source, and the straight line distance between the stations is $184.9 \mathrm{~m}$. While the photons are in flight, the electrical signal from the pump laser triggers a RNG, to choose a measurement setting. This occurs at a rate $79.3 \mathrm{MHz} / 800=99.1 \mathrm{KHz}$. At each station, the photons pass through an EOM and are detected by a superconducting nanowire single-photon detector (SNSPD) with a separately measured efficiency of $91 \%$ [29]. The detected signal is saved in a time tagger with a $10-\mathrm{MHz}$ clock. The process is completed before any information from the other station may arrive at luminal speed.

The EOMs remain on the same state for about $200 \mathrm{~ns}$, or 15 mode-locked pulses. Therefore, only the pulses at the center of this set fulfill the condition that the setting is completed before the photon arrival. In consequence, there is a compromise: taking into account only the central pulse No. 6 to get the best condition of spacelike separation, and scarce statistics, or else, adding neighboring pulses to improve the statistics at the cost of relaxing the condition of spacelike separation. The number of pulses taken into account into the statistics (which can be decided after the experiment has finished) is therefore a crucial parameter in this experiment. A table of $p$-values for different numbers of pulses and predictability excess of the RNG is provided. Excepting for the extreme cases, the memory loophole is clearly closed.

As in [5] (Sec. IV D), the probability per pulse of generating a pair is very low $\left(\approx 5 \times 10^{-4}\right)$, so that the chance of getting two events inside the same time window is negligible $(<1 \%)$. Yet, if the aggregate pulses do not fulfill the condition of being spacelike (even if less than one event per time window is recorded in the average) the test becomes, of course, unreliable.

The RNG in this setup deserves a special comment. There are three in each station. Two of them are relatively usual: One is based on measuring optical phase diffusion in a gain-switched laser, the other one on sampling the amplitude of an optical pulse at the single-photon level. The third one is unusual: it produces a bit by scrambling the digitized version of popular movies (one different in each station) with the digits of $\pi$. I had discussed the issue of the RNG in [11], and found that there were two possible sources of unpredictable results. One was a quantum state that projected into orthogonal bases. The other one was a series of random numbers stored in a computer. There was always an untestable hypothesis involved: (i) the projection of the quantum state was uncorrelated with the hidden variables carried by the pair, or (ii) the source of pairs was unable to read the memory of the computer. In this experiment, both possible sources are available and their outputs are scrambled. The predictability of this (in my opinion, "ultimate") source is estimated smaller than $\frac{1}{2}+10^{-4}=q_{\text {set }}$.

The value $J_{m}=1.41 \times 10^{-5}$ is obtained for five aggregate pulses (around the optimal pulse No. 6) more than three orders of magnitude smaller than the ideal value. The $p$ value is $5.9 \times 10^{-9}$, closing the memory loophole. The $p$ value obtained using the method developed in $[22,23]$ is comparable. If an excess predictability of the RNG even 15 times larger is allowed, the $p$ value rises to only $2.3 \times 10^{-7}$. The result for seven aggregate pulses is worse $\left(2 \times 10^{-7}\right.$ and $9.2 \times 10^{-6}$, respectively), in spite of fulfilling the spacelike condition and 
the improved statistics. This is probably due to instabilities of the voltage applied to the EOM near the moment a setting change is made.

The measured value of $P^{+}(a)$ is $2.17 \times 10^{-4}$, in the order of the value estimated in Sec. IV D and, once again, far from the ideal 0.081 . Then $q_{m}=\frac{1}{2}+1.6 \times 10^{-3}>q_{\text {set }}$ so that the locality loophole is closed. If the ideal value of $P^{+}(a)$ were used instead, $q_{m}=\frac{1}{2}+4.4 \times 10^{-5}$ and the loophole would not be closed.

The effect of background counts coming from blackbody radiation and room light is carefully taken into account. In order to reduce their number, the only events considered are those that occur within a window of 625 ps (at station A) and $781 \mathrm{ps}$ (B) around the natural time. The probability of observing a background count during the natural time is found to be $8.9 \times 10^{-7}$ (A) and $3.2 \times 10^{-7}$ (B). Yet, these small numbers suffice to raise $\eta_{\text {thr }}$ from $2 / 3$ up to 0.725 , marginally larger than $\eta_{\mathrm{eq}}=0.715$. Therefore, this experiment is in the limit of closing the detection loophole.

\section{F. Hensen et al. [7]}

This experiment is very different from the previously described ones. The entangled particles to be detected in this experiment are not photons, but the electronic spins associated with single nitrogen vacancy defects (NVs) in diamond chips. The spin orientation can be handled by applying rf signals. Its state can be measured with efficiency close to $100 \%$. The time required to measure the state of a NV is relatively long, which makes it necessary to have a distance between the stations longer than in the previous experiments, in order to ensure that all the setting choices and measurements are spacelike isolated. The NV are then placed in stations A at $493 \mathrm{~m}$ and B at 818 $\mathrm{m}$ from the "source" station (see below); the distance between A and B is $1280 \mathrm{~m}$. Each spin is entangled with an emitted photon, which is inserted into an optical fiber and sent to the source station. In this station the two photons are subjected to a Hong-Ou-Mandel measurement. If coincident photons are recorded at the two output ports of a beam splitter, then the biphoton state is $\left|\psi^{-}\right\rangle\left(\left|\psi_{E}\right\rangle\right.$ with $\left.r=-1\right)$ and then the two remote NVs are also in the state $\left|\psi^{-}\right\rangle$. This is because of the phenomenon of entanglement swapping, which is based on the following equality involving the states of the Bell's basis:

$$
\begin{aligned}
\left|\psi_{1 \mathrm{~A}}^{-}\right\rangle\left|\psi_{\mathrm{B} 2}^{-}\right\rangle= & \frac{1}{2}\left(\left|\psi_{12}^{+}\right\rangle\left|\psi_{\mathrm{AB}}^{+}\right\rangle-\left|\psi_{12}^{-}\right\rangle\left|\psi_{\mathrm{AB}}^{-}\right\rangle\right. \\
& \left.-\left|\phi_{\mathrm{AB}}^{+}\right\rangle\left|\phi_{\mathrm{AB}}^{+}\right\rangle+\left|\phi_{12}^{-}\right\rangle\left|\phi_{\mathrm{AB}}^{-}\right\rangle\right),
\end{aligned}
$$

where 1,2 are the photons and A,B are the NVs.

This setup is the closest to the original Bell's proposal, for the photons detected at the source not only prepare the NV state, but they also play the role of an "event-ready" signal heralding that an entangled state (of the two NVs) is available for measurement.

Yet the whole process occurs rarely. The probability of entanglement generation per attempt is estimated as $6.4 \times$ $10^{-9}$, or slightly more than one event-ready signal per hour. Once an event-ready signal from the source is recorded, the detection probability of the NV is almost $100 \%$, closing the detection loophole $\left(\eta_{\mathrm{thr}}=0.83\right.$ here). The whole experiment ran 245 trials during a total measurement time of $220 \mathrm{~h}$. The measured CHSH parameter is $S_{m}=2.42$ (it is noteworthy that it is higher than the previous estimation, $S_{\text {corr }}=2.30$ ). The HV + DZ model needs $q_{m}=0.659$ to fit this value. The fast RNGs are similar to the ones in [5] (Sec. IV D), but here the predictability excess is estimated as one order of magnitude smaller. Anyway, the precise numerical value of $q_{\text {set }}$ is irrelevant, for it is surely much smaller than $q_{m}$, so that the locality loophole is widely closed. The $p$ value is 0.039 , much larger than in $[5,6]$ (Secs. IV D and IV E) because of the smaller size of the statistics, but sufficient to close the memory loophole too.

\section{DISCUSSION AND CONCLUSIONS}

Table I shows that, leaving aside some details, the five experiments reach their goals. The models in [15-17], which study the consequences of a relaxation of measurement independence using approaches different from $\mathrm{HV}+\mathrm{DZ}$, produce also different values of $q_{m}$, but the essential results do not change, for the low level of predictability achieved in the experiments, especially in [7] (Sec. IV F), is widely sufficient to close the locality loophole for these approaches too.

In what follows, I discuss the details I find worth mentioning.

The reader may have perceived my admiration regarding the realization of the RNG, especially in the [6] (Sec. IVE). Yet, the condition $q_{\text {set }}<q_{m}$ is not fulfilled with a margin as wide as expected in the experiments using the EI. The cause of this weakness is not in the RNG, but in the low values of $J_{m}$ attained, which are from one to four orders of magnitude below the ideal. The same occurs with $P^{+}(a)$ excepting in [3] (Sec. IV B). Even though the locality loophole is closed according to the established criterion, the mentioned differences and the sensitivity of the results to numbers that are all close to zero leave a sense of uncertainty. The excellent value of $S$ obtained in [4] (Sec. IV C) with the same setup indicates that $\mathrm{CHSH}$ is more robust than EI and should be preferred, when possible. The reason why EI is chosen in [3-6] (Secs. IV B-IV E) is that the detectors' efficiencies are measured lower than the value of $\eta_{\text {thr }}$ for $\mathrm{CHSH}$.

The results of [7] (Sec. IV F) are more satisfactory because they do not merely violate the inequality $(S>2)$, but get halfway close to the ideal value $2 \sqrt{2}$. In order to achieve this remarkable result, the setup combines the advantages of propagating photons and the high detection efficiency of stationary NV spins. Entanglement swapping is used to teleport the photons' state to the spins. Nevertheless, entanglement swapping is a pure QM phenomenon with no classical or semiclassical counterpart. I wonder if a true logical loophole might be lurking there. For, in order to select the results of the NV measurements to be included into the statistically relevant set, one must assume QM correct, and QM certainly violates the Bell's inequalities. Perhaps what is believed to be demonstrated true by the observations is what is being assumed true from the data selection. This is a subtle issue that deserves to be studied in detail elsewhere.

The time-coincidence loophole was closed few years ago [30,31], but not simultaneously with the other loopholes. The experiment detailed in Sec. IV D [5] claims to have closed the detection and the time-coincidence loopholes together. Even 
though the setup is apparently able to reach this goal, the data analysis provided is, in my view, insufficient. A further analysis [32] uses the method developed in [22,23] and is based on the definition of a "distance" between time series to close the loophole, reaching a $p$ value smaller than $10^{-10}$. The achievements of this approach, including the detection of a fake source of entangled states, are remarkable. Yet, I find this approach more complex and indirect than the originally proposed one and, in consequence, more vulnerable to new loopholes. I believe that a "traditional" analysis of the time-tagged data in [5,6] (Secs. IV D and IV E), including the detections outside the natural time, is a more reliable way to close the time-coincidence loophole simultaneously with the others. As far as I know this analysis has not been done yet, but it may be done using the already recorded data.

Finally, [7] (Sec. IV F) is event-ready and hence is not affected by the time-coincidence loophole. Therefore, it closes all the loopholes without further analysis of the data.

Note that the thresholds have been calculated for each loophole separately. If the loopholes are combined (e.g, $q>\frac{1}{2}$ and $\eta<1$ ), the thresholds change depending on the details of the hidden variables model. It should also be kept in mind that more loopholes may be found in the future. Faking techniques, which have consequences for the security of QKD schemes, may be considered as a sort of new loophole [33]. It is therefore conceivable that an experiment entirely free of loopholes cannot be done [34]. What real experiments can do, in my opinion, is to shrink the space left to the loophole-based theories down to the point that (say, asymptotically) they become too exotic to be tenable. Also in my personal opinion, the reported experiments have already reached this point, and the defenders of LR should not insist on the little vulnerability left, but pay attention to some barely explored alternatives, for example, the possibility of nonergodic dynamics at the hidden variables level $[35,36]$.

During the process of review of this paper, another loophole-free and event-ready experiment was reported [37]. It uses the $\mathrm{CHSH}$ and is based on the measurement of the states of remote atoms linked through entanglement swapping, in a way similar to [7] (Sec. IV F). The value $S=2.22 \pm 0.033$ is obtained, hence violating the inequality, and a $p$ value $<10^{-9}$. These results confirm the conclusions already made.

Because of its purpose, this report must be critical with the claimed results. Yet I would like to emphasize my admiration for the extraordinary skills demonstrated by all the groups. They have developed new and formidable abilities of practical interest in the field of quantum information. They have also climbed many steps towards the ideal Bell's proposal. To say the least, they have imposed new and severe restrictions to the set of loophole-based theories.

\section{ACKNOWLEDGMENTS}

Many thanks to Scott Glancy and Lynden K. Shalm (NIST, Boulder) and to Yanbao Zhang (IQC, University of Waterloo) for their reading of the earlier versions of this paper, and for their interest, observations, and remarks. This contribution received support from Grant No. PIP11-077, Consejo Nacional de Investigaciones Científicas y Tecnológicas (CONICET), Argentina.

\section{APPENDIX: ORIGINAL HV + DZ MODEL}

Suppose the photons carry a hidden variable $\lambda$, similar to the classical plane of polarization, with a uniform distribution in $[0, \pi]$. If the source emits the totally symmetrical Bell state $\left|\phi^{+}\right\rangle$, the two photons of the pair carry the same value of $\lambda$. Let us also assume that the analyzers transmit (reflect) a photon with probability $=1$ if $0 \leqslant \lambda \leqslant \pi / 2(\pi / 2 \leqslant \lambda \leqslant \pi)$. In the $\lambda$ space, the analyzers have then two transmitting and two reflecting quadrants (see Fig. 1), and hence the total probabilities of transmission are $P^{+}(a)=P^{+}(b)=\frac{1}{2}$. The probability of coincidence $P^{++}(a, b)$ is given simply by the overlap of the two transmitting regions. Then $P^{++}(a, b)$ is a straight line between the points $\left\{a-b=0, P^{++}(a, b)=\frac{1}{2}\right\}$ and $\left\{a-b=\pi / 2, P^{++}(a, b)=0\right\}$. This produces $S_{\mathrm{CHSH}}=2$, exactly in the limit allowed by LR.

Let assume now that the pairs also carry a hidden variable $\mu$ with values 1 to 4 . It has the purpose of increasing $P^{++}$ for some settings (those having an angle between analyzers of $\pi / 8$ ) and to reduce it for others (the ones having $3 \pi / 8$ ). For example, if a photon with $\mu=1$ reaches the analyzer with setting $b$, the probability distribution on $\lambda$ is shifted an angle $\pi / 8$ "leftwards" (see Fig. 3 ) to increase $P^{++}\left(a^{\prime}, b\right)$ from $\frac{3}{8}$ to $\frac{1}{2}$ (which is its maximum possible value). There is no effect otherwise. In the same way, shifts are defined for the other three possible settings. Yet, the addition of $\mu$ is not enough to violate the inequality. The photons which miss their target produce an effect opposite to that desired and the average result is again $S_{\mathrm{CHSH}}=2$.

Let us assume now that the pairs with $\mu=1$ have a tendency to reach the setting $\left\{a^{\prime}, b\right\}, \mu=2$ to reach $\left\{a^{\prime}, b^{\prime}\right\}, \mu=3$ to

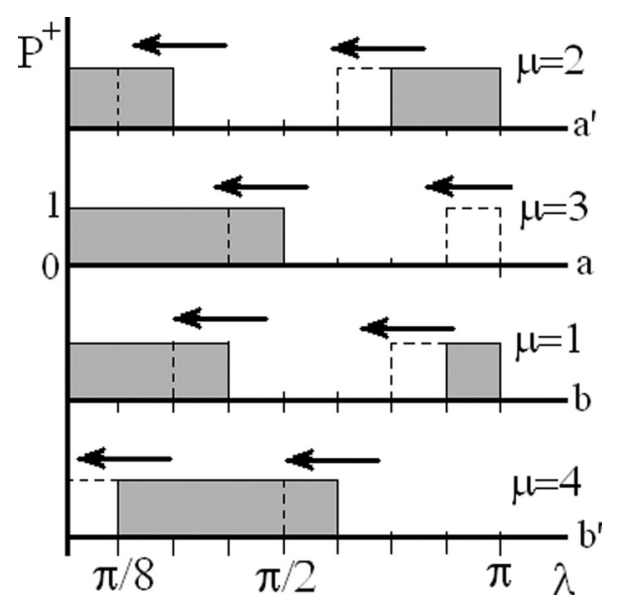

FIG. 3. Probability distributions in the original HV + DZ model (or $r=1$ case). The gray regions are the transmitting regions in the space of the hidden variable $\lambda$ for each setting. Note that they correspond simply to the angle orientation of the analyzers $\left\{a=0, a^{\prime}=-\pi / 4, b=-\pi / 8, b^{\prime}=\pi / 8\right\}$. The overlap of two gray regions gives the probability of coincidence [e.g., $P^{++}\left(a, b^{\prime}\right)=3 / 8$ ]. The arrows indicate the shifts (in an amount of $\pi / 8$ leftwards in all cases) of the regions in each setting when the additional hidden variable $\mu$ takes the indicated value. There is no shift otherwise. The shifted transmitting regions are indicated with dashed lines. Yet the shifts do not produce a violation of the inequality by themselves. Some correlation (measured with the parameter $q$ ) between the settings and $\mu$ is necessary to achieve it. 
reach $\{a, b\}$ and $\mu=4$ to reach $\left\{a, b^{\prime}\right\}$. The parameter $q$ (in [11] it was named $\frac{1}{2} \sqrt{a}$ ) is the probability a photon has to reach its target setting. The observable probability of coincidence of, e.g., $P^{++}\left(a^{\prime}, b\right)$ is (assuming $\mu$ is equally distributed)

$$
\begin{aligned}
P^{++}\left(a^{\prime}, b\right) & =q^{2} P^{\mu=1}\left(a^{\prime}, b\right)+q(1-q)\left[P^{\mu=2}\left(a^{\prime}, b\right)+P^{\mu=3}\left(a^{\prime}, b\right)\right]+(1-q)^{2} P^{\mu=4}\left(a^{\prime}, b\right) \\
& =q^{2} \frac{1}{2}+q(1-q)\left[\frac{1}{4}+\frac{3}{8}\right]+(1-q)^{2} \frac{3}{8} .
\end{aligned}
$$

For, if $\mu=1$, the target (which is $\left\{a^{\prime}, b\right\}$ in this case) is reached in both stations, if $\mu=2,3$ the target is reached in only one of the stations, and if $\mu=4$ both targets are missed. The other three coincidence probabilities are written in the same way. Yet, the resultant equations are linearly dependent; thus it suffices to ask that, e.g., $P^{++}\left(a^{\prime}, b\right)=\frac{1}{2} \cos ^{2}\left(a^{\prime}-b\right) \approx$ $0.4268 \Rightarrow q=0.769$ to exactly reproduce the $\mathrm{QM}$ predictions for all the settings.
[1] J. Clauser and A. Shimony, Bell's theorem: Experimental tests and implications, Rep. Prog. Phys. 41, 1881 (1978).

[2] J. Larsson, Loopholes in Bell inequality tests of local realism, J. Phys. A: Math. Theor. 47, 424003 (2014).

[3] M. Giustina, A. Mech, S. Ramelow, B. Wittman, J. Kofler, J. Beyer, A. Lita, B. Calkins, T. Gerrits, S. W. Nam et al., Bell violation using entangled photons without the fair-sampling assumption, Nature 497, 227 (2013).

[4] B. Christensen, K. T. McCusker, J. B. Altepeter, B. Calkins, T. Gerrits, A. E. Lita, A. Miller, L. K. Shalm, Y. Zhang, S. W. Nam et al., Detection-Loophole-Free Test of Quantum Nonlocality, and Applications, Phys. Rev. Lett. 111, 130406 (2013).

[5] M. Giustina, M. A. M. Versteegh, S. Wengerowsky, J. Handsteiner, A. Hochrainer, K. Phelan, F. Steinlechner, J. Kofler, J. A. Larsson, C. Abellan et al., A Significant Loophole-Free Test of Bell's Theorem with Entangled Photons, Phys. Rev. Lett. 115, 250401 (2015).

[6] L. Shalm, E. Meyer-Scott, B. G. Christensen, P. Bierhorst, M. A. Wayne, M. J. Stevens, T. Gerrits, S. Glancy, D. R. Hamel, M. S. Allman et al., A Strong Loophole-Free Test of Local Realism, Phys. Rev. Lett. 115, 250402 (2015).

[7] B. Hensen, H. Bernien, A. E. Dréau, A. Reiserer, N. Kalb, M. S. Blok, J. Ruitenberg, R. F. L. Vermeulen, R. N. Schouten, C. Abellán et al., Loophole-free Bell inequality violation using electron spins separated by 1.3 kilometres, Nature 526, 682 (2015).

[8] J. Clauser and M. Horne, Experimental consequences of objective local theories, Phys. Rev. D 10, 526 (1974).

[9] J. Larsson and R. Gill, Bell's inequality and the coincidence-time loophole, Europhys. Lett. 67, 707 (2004).

[10] P. H. Eberhard, Background level and counter efficiencies required for a loophole-free EPR experiment, Phys. Rev. A 47, R747(R) (1993).

[11] A. Hnilo, Hidden variables with directionalization, Found. Phys. 21, 547 (1991).

[12] A. Aspect, J. Dalibard, and G. Roger, Experimental Test of Bell's Inequalities Using Time-Varying Analyzers, Phys. Rev. Lett. 49, 1804 (1982).

[13] G. Weihs, T. Jennewein, C. Simon, H. Weinfurter, and A. Zeilinger, Violation of Bell's Inequality under Strict Einstein Locality Conditions, Phys. Rev. Lett. 81, 5039 (1998).
[14] A. Hnilo, A. Peuriot, and G. Santiago, Local realistic models tested by the EPRB experiment with random variable analyzers, Found. Phys. Lett. 15, 359 (2002).

[15] M. Hall, Local Deterministic Model of Singlet State Correlations, Phys. Rev. Lett. 105, 250404 (2010).

[16] J. Barrett and N. Gisin, How Much Measurement Independence is Needed in Order to Demonstrate Nonlocality? Phys. Rev Lett. 106, 100406 (2011).

[17] A. Bednorz and J. Zielinsky, The necessity of randomness in tests of Bell Inequalities, Phys. Lett. A 314, 362 (2003).

[18] D. Mermin, The best version of Bell's theorem, Ann. N. Y. Acad. Sci. 755, 616 (1995).

[19] J. Cereceda, Identification of all hardy-type correlations for two photons or particles with spin $\frac{1}{2}$, Found. Phys. Lett. 14, 401 (2001).

[20] A. E. Lita, A. J. Miller, and S. W. Nam, Counting near-IR single photons with 95\% efficiency, Opt. Express 16, 3032 (2008).

[21] R. Gill, Accardi contra Bell (cum mundi), the impossible coupling, arXiv:quant-ph/0110137.

[22] Y. Zhang, S. Glancy, and E. Knill, Asymptotically optimal data analysis for rejecting LR, Phys. Rev. A 84, 062118 (2011).

[23] Y. Zhang, S. Glancy, and E. Knill, Efficient quantification of experimental evidence against LR, Phys. Rev. A 88, 052119 (2013).

[24] P. Bierhorst, A rigorous analysis of the $\mathrm{CHSH}$ inequality experiment when trials need not be independent, Found. Phys. 44, 736 (2014).

[25] P. Bierhorst, A robust mathematical model for loophole-free Clauser-Horne experiments, J. Phys. A 48, 195302 (2015).

[26] J. Kofler, S. Ramelow, M. Giustina, and A. Zeilinger, On Bell violation using entangled photons without the fair-sampling assumption, arXiv:1307.6475.

[27] J. Kofler, M. Giustina, J.-Å. Larsson, and M. W. Mitchell, Requirements for a loophole-free photonic Bell test using imperfect setting generators, Phys. Rev. A 93, 032115 (2016).

[28] M. B. Agüero, A. A. Hnilo, and M. G. Kovalsky, Measuring the entanglement of photons produced by a pulsed source, J. Opt. Soc. Am. B 31, 3088 (2014).

[29] F. Marsili, V. B. Verma, J. A. Stern, S. Harrington, A. E. Lita, T. Gerrits, I. Vayshenker, B. Baek, M. D. Shaw, R. P. Mirin, and S. W. Nam, Detecting single infrared photons with $93 \%$ system efficiency, Nat. Photonics 7, 210 (2013). 
[30] M. B. Agüero, A. A. Hnilo, and M. G. Kovalsky, Time resolved measurement of the Bell's inequalities and the coincidenceloophole, Phys. Rev. A 86, 052121 (2012).

[31] J.-Å. Larsson, M. Giustina, J. Kofler, B. Wittman, R. Ursin, and S. Ramelow, Bell violation with entangled photons, free of the coincidence-time loophole, Phys. Rev. A 90, 032107 (2014).

[32] B. G. Christensen, A. Hill, P. G. Kwiat, E. Knill, S. W. Nam, K. Coakley, S. Glancy, L. K. Shalm, and Y. Zhang, Analysis of coincidence-time loopholes in experimental Bell tests, Phys. Rev. A 92, 032130 (2015).

[33] I. Gerhardt, Q. Liu, A. Lamas-Linares, J. Skaar, V. Scarani, V. Makarov, and C. Kurtsiefer, Experimentally Faking the
Violation of Bell's Inequalities, Phys. Rev. Lett. 107, 170404 (2011).

[34] R. D. Gill, Time, finite statistics, and Bell's fifth position, arXiv:quant-ph/0301059.

[35] V. Buonomano, A limitation on Bell's inequality, Ann. del'I. H. P., Sect. A 29, 379 (1978).

[36] A. Hnilo, On the meaning of an additional hypothesis in the Bell's inequalities, arXiv:1402.6177.

[37] W. Rosenfeld, D. Burchardt, R. Garthoff, K. Redeker, N. Ortegel, M. Rau, and H. Weinfurter, Event-ready Bell-test using entangled atoms simultaneously closing detection and locality loopholes, arXiv:1611.04604. 